\title{
Dermatomyositis as a symptom of primary lung cancer: A case report and review of the literature
}

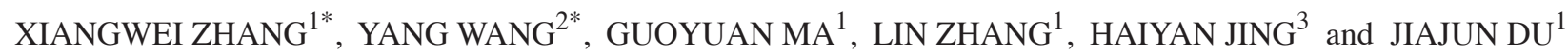 \\ Departments of ${ }^{1}$ Thoracic Surgery, ${ }^{2}$ Medical Imaging and ${ }^{3}$ Pathology, Shandong Provincial Hospital \\ Affiliated to Shandong University, Shandong University, Jinan, Shandong 250021, P.R. China
}

Received February 7, 2015; Accepted March 8, 2016

DOI: $10.3892 / 01.2016 .4418$

\begin{abstract}
Dermatomyositis (DM) is a rare autoimmune disease associated with manifestations of proximal muscle weakness and typical cutaneous skin rash. Patients with DM have a higher incidence rate of cancer, compared with the general population, since DM usually presents as a paraneoplastic syndrome. The present study reports a case of a 69 -year-old male patient presenting with typical cutaneous rash and aggravating proximal muscle weakness for 3 months. When first admitted to the Department of Dermatology of Shandong Provincial Hospital Affiliated to Shandong University (Jinan, China), the patient was diagnosed with DM. Subsequently, chest computed tomography scan revealed a neoplasm located in the right upper lobe. Video-assisted thoracoscopic surgery right upper lobectomy and lymph node dissection were then performed. The patient experienced a fast recovery from the surgery. The removal of the neoplasm clearly improved the symptoms of DM. Considering that DM is usually associated with the existence of a tumor, cases of DM require a thorough diagnostic investigation in order to exclude an underlying malignancy.
\end{abstract}

\section{Introduction}

Dermatomyositis (DM) is a rare, autoimmune disease of the connective, which is associated with immune complex deposition and subsequent inflammatory cell accumulation (1). The incidence rate of tumors in patients with DM has been reported to display a variable frequency of $0.6-1.0$ per 100,000 individuals (2). Diagnosis of dermatomyositis is associated with a higher risk of malignances, including the ovary, lung, pancreas, stomach, colorectal cancer and non-Hodgkin lymphoma

Correspondence to: Professor Jiajun Du, Department of Thoracic Surgery, Shandong Provincial Hospital Affiliated to Shandong University, Shandong University, 324 Jingwu Road, Jinan, Shandong 250021, P.R. China

E-mail: zhangxiangwei2011@126.com

${ }^{*}$ Contributed equally

Key words: primary lung cancer, dermatomyositis, surgery particularly for patients aged $>40$ years (3). The agents including corticosteroids and immunosuppressive drugs are always used in the treatment of dermatomyositis. The purposes of treatment are to improve the ability of daily living by increasing muscle strength and to alleviate extramuscular manifestations, including rash,dysphagia, dyspnoea, arthralgia and fever. Approximately $30 \%$ of the patients are left with mild to severe disability though the prognosis has been obviously improved. Age and association with tumor are risk factors associated with poor prognosis. DM often presents as a paraneoplastic syndrome, thus implying the presence of a tumor (4). A probable hypothesis for the development of DM is that shared immunological processes link malignancy with myopathy (5). The present study describes a case of DM and undifferentiated lung cancer, which was surgically treated. Following video-assisted thoracoscopic surgery (VATS) right upper lobectomy and lymph node dissection, the DM symptoms resolved.

\section{Case report}

A 69-year-old male patient, who was a former smoker (smoked 1 pack a day for 40 years), was admitted to the Shandong Provincial Hospital Affiliated to Shandong University (Jinan, China) in December 2010 presenting with typical cutaneous rash and aggravating proximal muscle weakness for 3 months. Three months prior to admission, the patient had noticed a rash of an unclear cause on his face, neck, chest, back and hands (Fig. 1). Almost at the same time, the patient experienced aggravating proximal muscle weakness. Upon admission to the hospital, laboratory test results revealed elevated levels of creatine kinase (CK) (627.7 U/l; normal values, 35.0-185.0 U/1). The results of immunological tests, including anti-double-stranded DNA (cat. no. EA1571; 1:200) and antinuclear (cat. no. EA1590-8; 1:100) antibody tests EUROIMMUN Medical Diagnostics (Beijing, China), were negative. The tumor markers carcinoembryonic antigen (cat. no. TF 3H8-1; 1:1), carbohydrate antigen (CA) 19-9 (cat. no. 121SLE; 1:1), CA 125 (cat. no. OC125; 1:1), alpha-fetoprotein (cat. no. ELA-04957; 1:1), neuron-specific enolase (cat. no. MRQ-55; 1:1), cytokeratin 19 fragment (cat. no. 11820966; 1:1) and prostate-specific antigen (cat. no. ER-PR8; 1:1) (monoclonal antibodies diluted with PBS and 10\% FBS, incubated for $30 \mathrm{~min}$ at room temperature; Roche Diagnostics, Shanghai, China) were all negative. Muscle strength testing revealed proximal muscle weakness. 


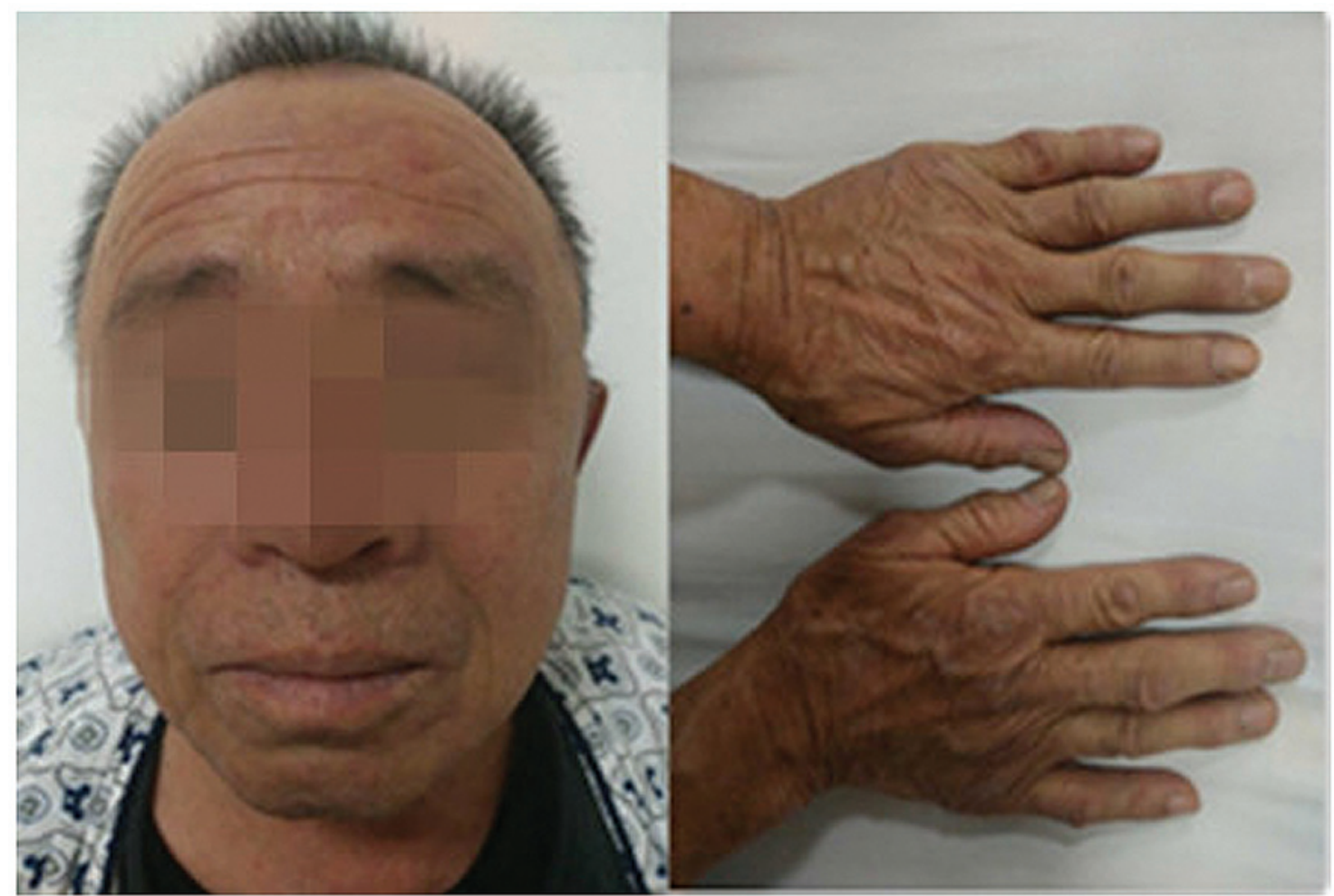

Figure 1. Typical skin rash on the face and hands of a patient with dermatomyositis.

A

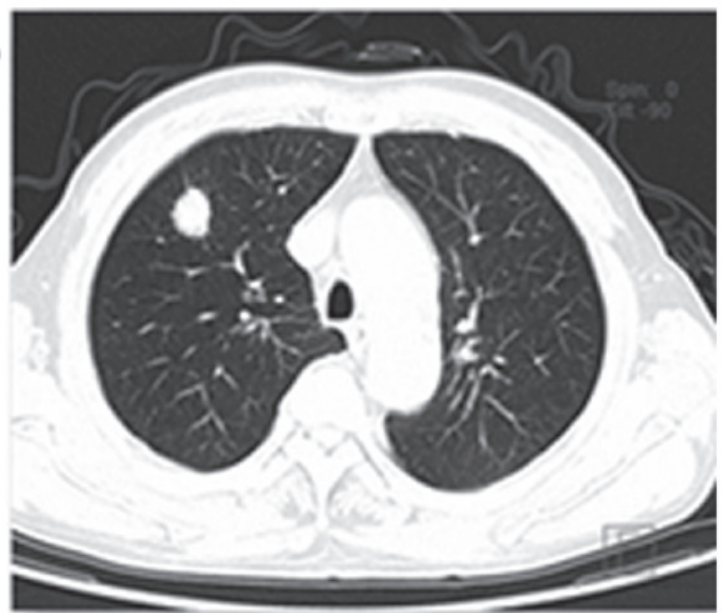

B

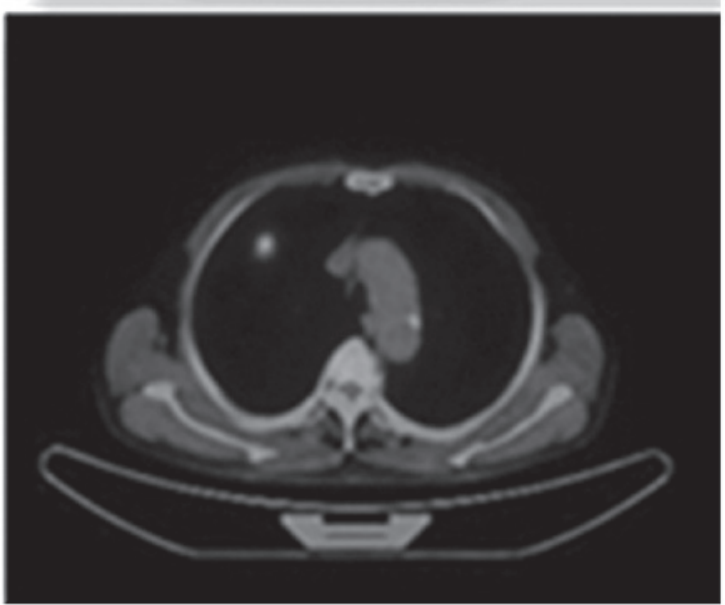

Figure 2. (A) Computed tomography scan showing a tumor located in the right upper lobe. (B) Positron emission tomography scan showing an abnormal fluorodeoxyglucose uptake by the tumor.

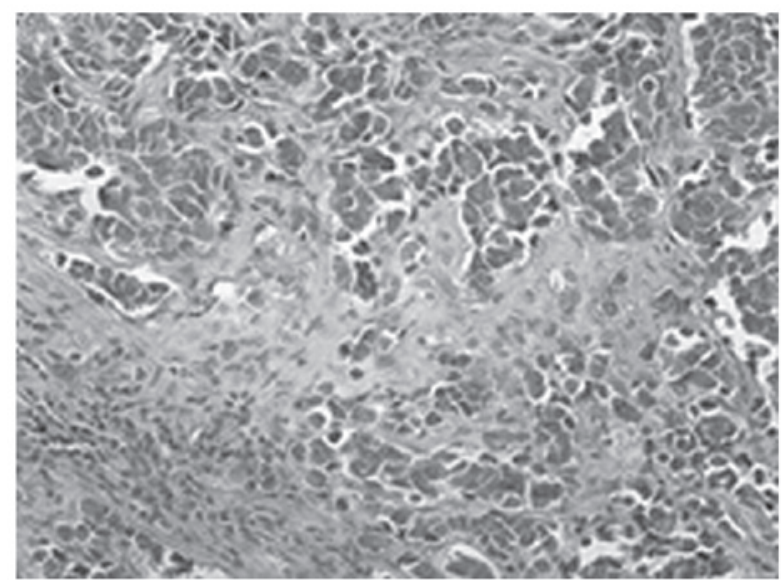

Figure 3. Postoperative pathology showing undifferentiated lung cancer (hematoxylin \& eosin staining; magnification, $\mathrm{x} 400$ ).

Electromyography performed with Keypoint 6 (9033A07; Dantec Dynamics, Skovlunde, Denmark) confirmed proximal myopathy. The patient was diagnosed with DM, based on the typical skin rash, elevated CK levels and abnormal electromyogram (Dantec Dynamics, Skovlunde, Denmark). Enhanced chest computed tomography (CT) scan (Siemens AG, Erlangen, Germany) revealed a tumor located in the right upper lobe. (Fig. 2A). Whole body positron emission tomography scan (GE Medical Systems, Waukesha, WI, USA) demonstrated a high fluorodeoxyglucose uptake rate by the tumor, with no obvious enlarged lymph nodes or distant metastasis (Fig. 2B). The final diagnosis was lung cancer (stage IB and T2aNOM0, based on the tumor-lymph-node-metastasis classification) (6), with DM presenting as a paraneoplastic syndrome. Upon diagnosis of 
$\mathrm{DM}$, the patient commenced treatment with $40 \mathrm{mg}$ oral prednisone (Qilu Pharmaceutical Co., Ltd., Jinan, China) and $200 \mathrm{mg}$ oral hydroxychloroquine (Sanofi, Shanghai, China) once per day, prior to being subjected to VATS (LTF-240, Olympus Medical Systems Corp., Tokyo, Japan) right upper lobectomy and lymph node dissection. Prednisone and hydroxychloroquine treatment was terminated $\sim 1$ month after surgery. Postoperative pathological examination using an Olympus Medical Systems Corp. microscope (BX51) confirmed undifferentiated cancer (Fig. 3) and pulmonary hilar lymph node metastasis.

The postoperative course of the patient was uneventful, and a considerable improvement in muscle weakness, as well as normalization of the CK levels, were observed. During the 6-month follow-up examination, the patient received chemotherapy and remained healthy, without DM symptoms or cancer relapse. However, the patient decided to discontinue the subsequent follow-ups in the Shandong Provincial Hospital Affiliated to Shandong University, and his clinical condition at the time of writing was unknown.

\section{Discussion}

$\mathrm{DM}$ is an idiopathic autoimmune disease characterized by skin and muscle lesions, with an incidence rate of 0.6-1.0 per 100,000 individuals (2) and a female to male ratio of 2:1 (1). DM mainly affects children and adults with two peak age ranges: Children aged 5-14 years and adults aged 45-60 years (1).

The exact disease mechanism has not been fully determined. DM is characterized by immune complex deposition in the capillary beds and frequent presence of inflammatory cell accumulation, mainly $\mathrm{T}$ cell-mediated myotoxicity or complement-mediated microangiopathy, which infiltrate myocytes leading to structural changes, including vacuolization and degeneration (2,7). Expression of capillary endothelium antigens, subsequent complement activation and immune complex deposition are known to induce endothelial cell edema, vacuolization, capillary necrosis, perivascular inflammation and ischemia, with muscle fiber destruction $(2,7)$.

Clinical symptoms of DM include progressive symmetric proximal muscle weakness and typical skin lesions (7). Serious cases of DM with respiratory muscle involvement may be fatal (8). The typical skin lesions associated with DM are heliotrope erythema around the eyes and dusky erythematous skin rashes on the face, neck, posterior arms and upper trunk (4). Other symptoms that may be present in different degrees include the following: i) Systemic symptoms, such as fever and weight loss; ii) musculoskeletal changes, such as arthralgia and synovitis; iii) gastrointestinal disorders, such as dysphagia, dysmotility and malabsorption; iv) cardiac and pulmonary disorders, such as tachyarrhythmia and interstitial lung disease; and v) vascular disorders, such as Raynaud's phenomenon and vasculitis (9).

DM diagnosis is mainly based on the Bohan and Peter criteria listed below: i) Progressive proximal and symmetric muscle weakness; ii) muscle biopsy exhibiting characteristic changes of myositis; iii) elevated levels of serum skeletal muscle enzymes; iv) electromyography indicating primary myopathic changes; and v) typical skin lesions (10). In the present case, the diagnosis of DM was based on the above symptoms iii-v.

An association between DM and an increased risk of malignancy has been previously demonstrated. Tumors have been shown to usually occur following the onset of DM, or simultaneously with it $(3,11)$. The most common malignancies associated with DM are lung, colorectal, cervical and ovarian cancer (12); however, the cause and effect relationship between cancer and $\mathrm{DM}$ is not fully understood (4). Several possible hypotheses have been established regarding the occurrence of DM and malignancy-immune disorders, viral infections or substance secretion by cancer cells that facilitate abnormal immunologic responses. The tumors may share cross-reacting antigens with muscle fibers, tendon sheath and blood vessels. Related cross-reacting antibodies may lead to concurrent occurrence of DM (13). In numerous cases, DM manifests as a paraneoplastic syndrome. The paraneoplastic clinical symptoms not directly associated with the primary tumor or metastasis have been shown to resolve following complete removal of the tumor (3); in case of tumor relapse, muscle weakness and typical skin rash recurrence is observed, suggesting the paraneoplastic nature of DM (14). There also appear to be some clinical differences in these patients, as their CK values are closer to normal and are less likely to present myositis-specific antibodies (11).

In the current case, DM presented as a paraneoplastic syndrome accompanied by undifferentiated cancer of the right upper lobe. DM symptoms comprised the first indication of cancer. Surgical removal of the tumor was performed, which led to a marked alleviation of the symptoms, thus supporting the paraneoplastic nature of DM.

In conclusion, DM is a disease with a relatively simple clinical diagnosis; however, clinicians consider the possible association of DM with malignancy. The existence of occult primary tumors should be excluded in all patients with a diagnosis of DM, based on patient's age, gender, local epidemiology and risk factors. Patients aged $>40$ years should be particularly screened for potential malignancies. The results of the present study suggested that early diagnosis and timely treatment is crucial for the prognosis of the tumor. In addition, a diagnosis of DM should always be considered a warning sign of malignancy.

\section{References}

1. Mastaglia FL and Phillips BA: Idiopathic inflammatory myopathies: Epidemiology, classification, and diagnostic criteria. Rheum Dis Clin North Am 28: 723-741, 2002.

2. Dalakas MC and Hohlfeld R: Polymyositis and dermatomyositis. Lancet 362: 971-982, 2003.

3. Hill CL, Zhang Y, Sigurgeirsson B, Pukkala E, Mellemkjaer L, Airio A, Evans SR and Felson DT: Frequency of specific cancer types in dermatomyositis and polymyositis: A population-based study. Lancet 357: 96-100, 2001.

4. Callen JP: Relation between dermatomyositis and polymyositis and cancer. Lancet 357: 85-86, 2001.

5. Buchbinder R and Hill CL: Malignancy in patients with inflammatory myopathy. Curr Rheumatol Rep 4: 415-426, 2002.

6. Detterbeck FC, Boffa DJ and Tanoue LT: The new lung cancer staging system. Chest 136: 260-271, 2009.

7. Dalakas MC: Inflammatory disorders of muscle: Progress in polymyositis, dermatomyositis and inclusion body myositis. Curr Opin Neurol 17: 561-567, 2004.

8. Fathi M, Lundberg IE and Tornling G: Pulmonary complications of polymyositis and dermatomyositis. Semin Respir Crit Care Med 28: 451-458. 2007. 
9. Dalakas MC: Polymyositis, dermatomyositis and inclusion-body myositis. N Engl J Med 325: 1487-1498, 1991.

10. Miller FW, Rider LG, Plotz PH, Isenberg DA and Oddis CV: Diagnostic criteria for polymyositis and dermatomyositis. Lancet 362: 1762-1763, 2003.

11. Chow WH, Gridley G, Mellemkjaer L, McLaughlin JK, Olsen JH and Fraumeni JF Jr: Cancer risk following polymyositis and dermatomyositis: A nationwide cohort study in Denmark. Cancer Causes Control 6: 9-13, 1995.

12. Fujita J, Tokuda M, Bandoh S, Yang Y, Fukunaga Y, Hojo S, Ueda Y,Dobashi N, Dohmoto K, Ishida T and Takahara J: Primary lung cancer associated with polymyositis/dermatomyositis, with a review of the literature. Rheumatol Int 20: 81-84, 2001.
13. Andras C, Ponyi A and Constantin T: Dermatomyositis and polymyositis associated with malignancy: A 21-year retrospective study. J Rheumatol 35: 438-444, 2008.

14. Zang YS, Xiu QY, Fang Z, Li B and Xia TB: Case report: Dramatic recovery of lung adenocarcinoma-associated dermatomyositis with targeted lung cancer therapy alone. Oncologist 13: 79-81, 2008. 